\title{
RBF Neural Network Sliding Mode Control Method Based on Backstepping for an Electro-hydraulic Actuator
}

\author{
Wending $\mathrm{Li}^{1,2,3,{ }^{*}}$ - Guanglin Shi ${ }^{1}$ - Chun Zhao 2,3 - Hongyu Liu ${ }^{2,3}$ - Junyong Fu ${ }^{2,3}$ \\ ${ }^{1}$ Shanghai Jiao Tong University, School of Mechanical Engineering, China \\ ${ }^{2}$ Shanghai Aerospace Control Technology Institute, China \\ ${ }^{3}$ Shanghai Servo-system Engineering Research Center, China
}

\begin{abstract}
Aiming at the interference problem and the difficulty of model parameter determination caused by the nonlinearity of the valve-controlled hydraulic cylinder position servo system, this study proposes a radial basis function (RBF) neural network sliding mode control strategy based on a backstepping strategy for the electro-hydraulic actuator. First, the non-linear system model of the third-order position electro-hydraulic control servo system is established on the basis of the principle analysis. Second, the model function RBF adaptive law and backstepping control law are designed according to Lyapunov's stability theorem to solve the problem of external load disturbance and modelling uncertainty, combined with sliding mode control strategy and virtual control law. Finally, simulation and experiment on MATLAB Simulink and semi-physical experimental platform are accomplished to show the effectiveness of the proposed method. Moreover, results show that the designed controller has high tracking accuracy to the given signal.
\end{abstract}

Keywords: RBF neural network, sliding mode, backstepping, non-linear control, electro-hydraulic actuator

\section{Highlights}

- The third-order non-linear model of electro-hydraulic servo system is established with disturbance.

- The observer and the sliding mode term are used to remove the different disturbances.

- By introducing the backstepping method, the virtual control quantity is solved first, and then the control law is solved by the neural network sliding mode algorithm, which achieves high control precision.

- The simulation analysis of high-speed and low-speed operating conditions was carried out, and the experimental results were compared with the simulation results, which showed good consistency.

\section{INTRODUCTION}

The electro-hydraulic servo control system is widely used in aerospace, national defence, civil industry, robot [1], and other fields because of its large powerweight ratio, rapid response speed, strong bearing capacity, and high control accuracy [2]. Electrohydraulic servo actuators are often used as the execution unit, such as the thrust vector control of rockets and missiles, attitude control systems of aircraft and tanks, stability control systems of vehicle active suspension, and control systems of mill presses [3]. As a key executive system, the performance of the electro-hydraulic servo actuator directly influences the precision, stability, and reliability of the control system [4]. The requirements of the electro-hydraulic control system are also continually improved in terms of mechanical working accuracy [5], response speed, and automation degree [6]. This improvement requires not only the high performance of hydraulic control components but also involves the use of advanced control strategies. However, the high-performance requirement of electro-hydraulic servo system (e.g., complexity, nonlinearity, parameter uncertainty, and load variability) cannot be achieved by using the traditional control methods. The use of intelligent control methods in solving the control problem of the electro-hydraulic servo system is a key technical issue in the development of high-performance electrohydraulic servo systems [7].

In recent years, many scholars have applied advanced control methods, such as adaptive, robust, sliding mode, and intelligent controls [8], to the electro-hydraulic servo control system to improve the control performance of the system, and have achieved varied results [9]. However, the work environment of the electro-hydraulic servo system of a launch vehicle is complex, that is, the load characteristics have many new characteristics, and the stability of the rocket control system requires the launch vehicle to have good dynamic characteristics [10]. Therefore, other effective control strategies need to be explored [11]. The backstepping method, which refers to a step-bystep method of designing control laws, is effective in solving non-linear control problems [12]. Moreover, many scholars have investigated and applied this method in non-linear system control [13]. Neural network control is a new strategy in overcoming the control problem of systems that are complex, nonlinear, or uncertain [14]. Sliding mode control is a 
special kind of non-linear control that designs the switching hyperplane of the system according to the expected dynamic characteristics of the system [15].

In the present study, a three-order sliding mode control of the neural network method of the radial basis function (RBF) is proposed on the basis of the backstepping strategy for the servo system of valvecontrolled electro-hydraulic position to solve further the non-linear modelling uncertainty and interference of the combined neural network, sliding mode, and backstepping technologies [16].

\section{NON-LINEAR MATHEMATICAL MODEL AND PROBLEM DESCRIPTION}

In this study, the valve-controlled electro-hydraulic servo system is mainly composed of a controller, a motor pump power source, an accumulator, a pressure sensor, a relief valve, a servo valve, a hydraulic lock, a position sensor, and a hydraulic cylinder [17], as shown in Fig. 1.

(1) Motor
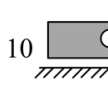

(2) Pumb

(3) Accumulator

(4) Pressure sensor

(5) Relief valve

(6) Servo valve

(7) Hydraulic lock

(8) Position sensor

(9) Hydraulic cylinder

(10)Load

(11)Tank

Fig. 1. The electro-hydraulic actuator system

The system block diagram of the electrohydraulic servo system is shown in Fig. 2.

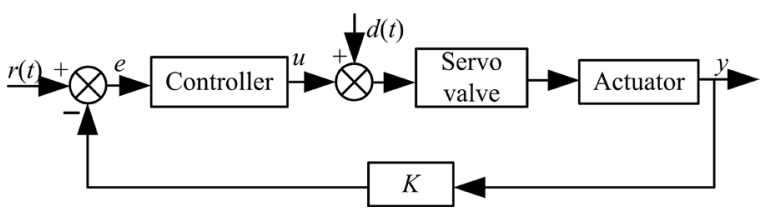

Fig. 2. Block diagram of the electro-hydraulic actuator

In Fig. 2, $r(t)$ is the reference input or set value of the system, $e$ is the control deviation, $u$ is the control law, $y$ is the system output, $d(t)$ is the continuous external disturbance, and $k$ is the feedback coefficient.
By measuring the feedback signal in the position, a closed loop of the negative feedback deviation control is formed to meet the requirements of the output signal.

The kinematic equation of the system is determined by considering the non-linear factors.

$$
m \ddot{y}=p_{L} A-B \dot{y}-A_{f} S_{f}(\dot{y})+f(y, \dot{y}, t),
$$

where $m$ is equivalent to the total weight of the load on the piston rod, $y$ is the piston rod output displacement, $p_{L}$ is the pressure difference between two cavities of the actuator, $A$ is the area of action of the liquid pressure, $B$ is the viscous damping coefficient, $A_{f}$ is the Coulomb friction amplitude, $S_{f}(\dot{y})$ is the Coulomb friction shape function, and $f(y, \dot{y}, t)$ is the unmodeled dynamic and external disturbance function.

The pressure dynamic equations of the actuator are expressed as follows [18]:

$$
\begin{gathered}
\dot{p}_{1}=\frac{\beta}{V_{01}+A y}\left(-A \dot{y}-C_{t} p_{L}+Q_{1}\right), \\
\dot{p}_{2}=\frac{\beta}{V_{02}-A y}\left(A \dot{y}+C_{t} p_{L}-Q_{2}\right), \\
Q_{1}=\sqrt{2} k_{q 1} x_{v}\left[\Theta\left(x_{v}\right) \sqrt{p_{s}-p_{1}}+\Theta\left(-x_{v}\right) \sqrt{p_{1}-p_{r}}\right],
\end{gathered}
$$

where $p_{1}$ and $p_{2}$ are the pressure values of the two cavities of the actuator, $V_{01}$ and $V_{02}$ are the initial volumes of the two cavities of the actuator, $\beta$ is the bulk modulus of elasticity of oil, $C_{t}$ is the leakage coefficient, and $Q_{1}$ and $Q_{2}$ are the flow rates of the two cavities of the actuator.

The flow equation of the servo valve to the twocavity actuator is presented as follows:

$$
\begin{aligned}
& Q_{1}=\sqrt{2} k_{q 1} x_{v}\left[\Theta\left(x_{v}\right) \sqrt{p_{s}-p_{1}}+\Theta\left(-x_{v}\right) \sqrt{p_{1}-p_{r}}\right], \\
& Q_{2}=\sqrt{2} k_{q 2} x_{v}\left[\Theta\left(x_{v}\right) \sqrt{p_{2}-p_{r}}+\Theta\left(-x_{v}\right) \sqrt{p_{s}-p_{2}}\right],
\end{aligned}
$$

where $k_{q 1}$ and $k_{q 2}$ are the servo valve flow gains, $x_{v}$ is the servo valve spool opening, $p_{s}$ is the oil inlet pressure, and $p_{r}$ is the oil outlet pressure. The value of $\Theta(\cdot)$ can be expressed as follows:

$$
\Theta(\cdot)=\left\{\begin{array}{ll}
1, & \cdot \geq 0 \\
0, & \cdot<0
\end{array} .\right.
$$

Assume that $k_{q}=k_{q 1}=k_{q 2}$. The dynamic servo valve can be simplified to a proportional link because the frequency width of the servo valve is remarkably higher than that of the system, $x_{v}=k_{i} u$. In this case, $\Theta\left(x_{v}\right)=\Theta(u)$. 
If $k_{u}=\sqrt{2} k_{q} k_{i}$,

and $\Lambda_{1}=\left[\Theta(u) \sqrt{p_{s}-p_{1}}+\Theta(-u) \sqrt{p_{1}-p_{r}}\right]$,

and $\Lambda_{2}=\left[\Theta(u) \sqrt{p_{2}-p_{r}}+\Theta(-u) \sqrt{p_{s}-p_{2}}\right]$

then the flow equation can be expressed as:

$Q_{1}=k u \Lambda_{1} u, Q_{2}=k u \Lambda_{1} u$

By combining Eqs. (2) and (3), we get

$$
\begin{aligned}
\dot{p}_{1}-\dot{p}_{2}= & \left(\frac{\Lambda_{1}}{V_{1}}+\frac{\Lambda_{2}}{V_{2}}\right) k_{u} \beta u-\left(\frac{1}{V_{1}}+\frac{1}{V_{2}}\right) A \beta x_{2} \\
& -\left(\frac{1}{V_{1}}+\frac{1}{V_{2}}\right) \beta C_{t} p_{L} .
\end{aligned}
$$

Set the state variables to $x_{1}=y, x_{2}=\dot{y}$, and $x_{3}=y\left(p_{1}-p_{2}\right) A=p_{L} A$, and the state equation of the electro-hydraulic servo actuator is expressed as follows:

$$
\left\{\begin{array}{rl}
\dot{x}_{1} & =x_{2} \\
\dot{x}_{2} & =\frac{1}{m} x_{3}-\frac{1}{m} B x_{2}-\frac{1}{m} A_{f} S_{f}\left(x_{2}\right)+\frac{1}{m} f(y, \dot{y}, t) \\
\dot{x}_{3}= & \left(\frac{\Lambda_{1}}{V_{1}}+\frac{\Lambda_{2}}{V_{2}}\right) k_{u} \beta u-\left(\frac{1}{V_{1}}+\frac{1}{V_{2}}\right) A^{2} \beta x_{2} \\
& -\left(\frac{1}{V_{1}}+\frac{1}{V_{2}}\right) \beta C_{t} p_{L}+d_{2}(t)
\end{array} .\right.
$$

To simplify the expression, let

$$
f_{1}(x)=\frac{1}{m} B x_{2}+\frac{1}{m} A_{f} S_{f}\left(x_{2}\right),
$$

$g(u, x)=\left(\frac{\Lambda_{1}}{V_{1}}+\frac{\Lambda_{2}}{V_{2}}\right) k_{u} A \beta$, and

$f_{2}(x)=\left(\frac{1}{V_{1}}+\frac{1}{V_{2}}\right) A^{2} \beta x_{2}+\left(\frac{1}{V_{1}}+\frac{1}{V_{2}}\right) \beta C_{t} p_{L}$, and

$d_{1}(t)=\frac{f_{1}\left(x_{1}, x_{2}, t\right)}{m}$.

Then, Eq. (8) has the following form:

$$
\left\{\begin{array}{l}
\dot{x}_{1}=x_{2} \\
\dot{x}_{2}=\frac{1}{m} x_{3}-f_{1}(x)+d_{1}(t) \\
\dot{x}_{3}=g(u, x) u-f_{2}(x)+d_{2}(t)
\end{array} .\right.
$$

Eq. (9) shows that strong nonlinearity exists, and building an accurate model of the system is difficult. At the same time, the external interference has a substantial influence on the system.

\section{STRATEGY SELECTION AND CONTROLLER DESIGN}

\subsection{Strategy Selection}

The backstepping method is an effective non-linear control method that emerged in the 1990s [19]. This method can solve the problem of the lack of constructivism in the traditional Lyapunov function [20]. According to the structural characteristics of the controlled object, the backstepping method establishes the Lyapunov function of the entire system via progressive recursion and realizes the stability control of the non-linear closed-loop system [21].

The RBF neural network algorithm can approximate any non-linear network [22], and the sliding mode control algorithm can improve the robustness of the system by selecting switching functions [23]. The RBF neural network is a kind of a single hidden layer of the three-layer feedforward network with input and output non-linear mappings. The network structure is shown in Fig. 3.

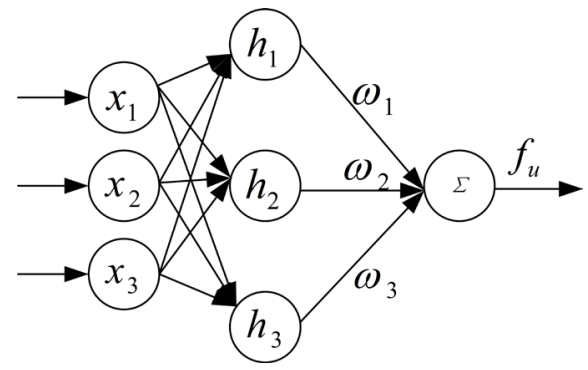

Fig. 3. RBF neural network structure

$$
\begin{aligned}
& h_{j}=\exp \left(-\frac{\left\|\mathbf{x}-\mathbf{c}_{j}\right\|_{2}^{2}}{2 b_{j}^{2}}\right), \\
& f_{u}=\mathbf{W}^{* \mathrm{~T}} \mathbf{h}_{f}(x)+\varepsilon_{f},
\end{aligned}
$$

where $f_{u}$ is the ideal RBF network output, $\mathbf{x}$ is the RBF network input, $j$ is the $j^{\text {th }}$ node of the network hidden layer, $\mathbf{h}=\left[h_{j}\right]^{\mathrm{T}}$ is the Gaussian basis function output of the network, $\mathbf{W}^{*}$ is the ideal weight of the network, $b_{j}$ is the base width parameter of node $j, b_{j}>0, c_{j}$ is the centre vector of the network node $j$, and $\varepsilon_{f}$ is the network approximation error.

Sliding mode control is a discontinuous kind of non-linear control method for designing a stable motion mode, that is, the sliding mode surface for the system state or error in advance, and creating the controller to guide the system trajectory to this preset mode and force the system trajectory to maintain and move along the mode in the future [24]. The system is defined as $x=f(x, u, t)$ if the plane of state $s(x)$ and 
control function $u(x)$ are available. The sliding mode control can be expressed as follows:

$$
u=\left\{\begin{array}{l}
u^{+}(x), s(x)>0 \\
u^{-}(x), s(x)>0
\end{array} u^{+}(x) \neq u^{-}(x) .\right.
$$

The system triggered from any initial state can reach the state plane $s(x)=0$ within a certain period, and the steady-state point of the system is reached under the action of $u(x)$.

\subsection{Controller Design}

A state observer of disturbance $d_{1}(t)$ is designed according to the method in [25], $x_{e}=d_{1}(t)$.

$$
\left\{\begin{array}{l}
\dot{\hat{x}}_{1}=\hat{x}_{2}+3 \omega_{e}\left(x_{1}-\hat{x}_{1}\right) \\
\dot{\hat{x}}_{2}=\frac{x_{3}}{m}-f_{1}(x)+x_{e}+3 \omega_{e}^{2}\left(x_{1}-\hat{x}_{1}\right), \\
\dot{\hat{x}}_{e}=\omega_{e}^{3}\left(x_{1}-\hat{x}_{1}\right)
\end{array}\right.
$$

$\tilde{d}_{1}(t)$ is the observation error. Eq. (9) can be written as follows:

$$
\left\{\begin{array}{l}
\dot{x}_{1}=x_{2} \\
\dot{x}_{2}=\frac{1}{m} x_{3}-f_{1}(x)+\hat{d}_{1}(t)+\tilde{d}_{1}(t) . \\
\dot{x}_{3}=g(u, x) u-f_{2}(x)+d_{2}(t)
\end{array}\right.
$$

The state error of the system is defined as:

$$
\left\{\begin{array}{l}
e_{1}=x_{1}-x_{1 d} \\
e_{2}=\dot{e}_{1}+k_{1} e_{1}=x_{2}-x_{2 d}, \\
e_{3}=\dot{e}_{2}+k_{2} e_{2}=x_{3}-\alpha
\end{array}\right.
$$

where, $x_{1 d}$ is the input of the system, $x_{2 d}=x_{1 d}-k_{1} e_{1}$, and $\alpha$ is the virtual control quantity of $x_{3}$. The error can be written in this form to avoid differential explosion.

1. Step 1: Find the virtual control law [26]

The sliding mode surface is defined as Eq. (16):

$$
s_{1}=c_{e 1} e_{1}+e_{2}
$$

Substituting Eqs. (14) and (15) into Eq. (16):

$$
\dot{s}_{1}=c_{e 1} \dot{e}_{1}+\frac{1}{m} x_{3}-f_{1}(x)+\hat{d}_{1}(t)+\tilde{d}_{1}(t)-\dot{x}_{2 d} .
$$

An appropriate positive real number $\eta_{1}$ is selected to design the control law based on disturbance observation and sliding mode. The virtual control quantity $\alpha$ is:

$$
\begin{aligned}
& \alpha=-m\left(c_{e 1} \dot{e}_{1}-f_{1}(x)-\dot{x}_{2 d}+\hat{d}_{1}(t)+\mu s_{1}\right) \\
& -\eta_{1} m \operatorname{sgn}\left(s_{1}\right) .
\end{aligned}
$$

In order to alleviate the discontinuity of sliding mode switching and solve the problem of indifferentiability of sign function, the sign function $\operatorname{sgn}(s)$ is replaced by $2 / \pi \arctan (K s) . K$ is a positive real number, and the greater the value, the more accurate the approximation. When $K=300$, the approximate curve of sign function represented by $2 / \pi \arctan (K s)$ is shown in Fig. 4.

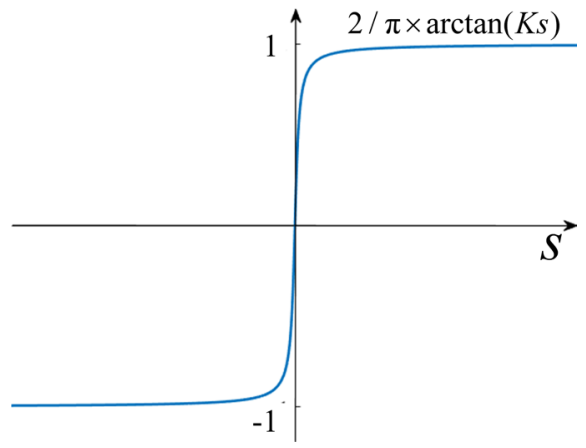

Fig. 4. Approximation curve of the sign function

The virtual control rate $\alpha$ can be expressed as:

$$
\begin{aligned}
\alpha & =-m\left(c_{e 1} \dot{e}_{1}-f_{1}(x)-\dot{x}_{2 d}+\hat{d}_{1}(t)+\mu s_{1}\right) \\
& -\frac{2}{\pi} \times \eta_{1} m \arctan \left(K_{1} s_{1}\right),
\end{aligned}
$$

where, $K_{1}$ is a sufficiently large positive real number.

The Lyapunov function is defined as [27]:

$$
\begin{gathered}
V_{1}=\frac{1}{2} s_{1}^{2}, \\
\dot{s}_{1}=-\mu s_{1}-\frac{\pi}{2} \times \eta_{1} \arctan \left(K_{1} s_{1}\right)+\tilde{d}_{1}(t), \\
\dot{V}_{1}=-\mu s_{1}^{2}-\frac{2}{\pi} \eta_{1} s_{1} \arctan \left(K_{1} s_{1}\right)+s_{1} \tilde{d}_{1}(t) .
\end{gathered}
$$

When $s_{1} \in(-\infty, \infty)$, it can be proved that:

$$
\frac{\pi}{2}\left|s_{1}\right|-\frac{1}{K_{1}} \leq s_{1} \arctan \left(K_{1} s_{1}\right) \leq \frac{\pi}{2}\left|s_{1}\right| .
$$

According to Eqs. (22) and (23), the following equation can be obtained:

$$
\begin{aligned}
\dot{V}_{1} & \leq-\mu s_{1}^{2}-\eta_{1}\left|s_{1}\right|+\frac{2}{\pi K_{1}} \eta_{1}+s_{1} \tilde{d}_{1}(t) \\
& \leq-\mu s_{1}^{2}+\frac{2}{\pi K_{1}} \eta_{1} .
\end{aligned}
$$

According to the method in reference [28], the inequality in Eq. (24) can be solved as follows: 


$$
V_{1}(t) \leq \frac{\eta_{1}}{\pi K_{1} \mu} .
$$

$V_{1}(t)$ converges asymptotically, and the law of convergence is affected by the values of $\eta_{1}, K_{1}$ and $\mu$.

2. Step 2: Find the system control law

Definition of sliding surface:

$$
s_{2}=d_{e 1} e_{1}+d_{e 2} e_{2}+e_{3}
$$

The derivative of Eq. (26) is:

$$
\dot{s}_{2}=d_{e 1} \dot{e}_{1}+d_{e 2} \dot{e}_{2}+g(u, x) u-f_{2}(x)+d_{2}(t)-\dot{\alpha} \text {. }
$$

Design control law $u$ :

$$
\begin{aligned}
u= & \frac{1}{g(u, x)}\left[-d_{e 1} \dot{e}_{1}-d_{e 2} \dot{e}_{2}+f_{2}(x)\right. \\
& \left.+\dot{\alpha}-\eta_{2}^{\prime} \arctan \left(K_{2} s_{2}\right)\right] .
\end{aligned}
$$

Due to the existence of nonlinearity, the mathematical models of $f_{2}(x)$ and $g(u, x)$ cannot be accurately obtained and the control law cannot be solved. Therefore, $f_{2}(x)$ and $g(u, x)$ need to be estimated or approximated. RBF neural network learning method is adopted to approximate functions $f_{2}(x)$ and $g(u, x)$, then:

$$
\left\{\begin{array}{l}
g(u, x)=\mathbf{W}_{g}^{*} \mathbf{h}_{g}(x)+\varepsilon_{g} \\
f_{2}(x)=\mathbf{W}_{f 2}^{*} \mathbf{h}_{f}(x)+\varepsilon_{f}
\end{array} .\right.
$$

$\widehat{\mathbf{W}}_{g}$ and $\widehat{\mathbf{W}}_{f 2}$ are respectively the dynamic estimated weights of the neural network approximation of function $g(u, x)$ and $f_{2}(x)$. The neural network approximation of $g(u, x)$ and $f_{2}(x)$ is written in the following form:

$$
\left\{\begin{array}{l}
\hat{g}(u, x)=\widehat{\mathbf{W}}_{g} \mathbf{h}_{g}(x) \\
\widehat{f}_{2}(x)=\widehat{\mathbf{W}}_{f_{2}} \mathbf{h}_{f}(x)
\end{array} .\right.
$$

The control law, Eq. (28), is written as follows:

$$
\begin{aligned}
u= & \frac{1}{\hat{g}(u, x)}\left[-d_{e 1} \dot{e}_{1}-d_{e 2} \dot{e}_{2}+\widehat{f}_{2}(x)\right. \\
& \left.+\dot{\alpha}-\eta_{2}^{\prime} \arctan \left(K_{2} s_{2}\right)\right] .
\end{aligned}
$$

The observation errors are: $\tilde{g}(u, x), \tilde{f}_{2}(x)$,

$$
\begin{gathered}
g(u, x)=\hat{g}(u, x)-\tilde{g}(u, x), \\
f_{2}(x)=\widehat{f}_{2}(x)-\tilde{f}_{2}(x),
\end{gathered}
$$

Substitute Eq. (32) into Eq. (27):

$$
\begin{aligned}
\dot{s}_{2}= & d_{e 1} \dot{e}_{1}+d_{e 2} \dot{e}_{2}-\tilde{g}(u, x) u \\
& +\hat{g}(u, x) u-f_{2}(x)+d_{2}(t)-\dot{\alpha},
\end{aligned}
$$

where, $\dot{\alpha}=-m\left\{c_{e 1} \ddot{e}_{1}+\dot{f}_{1}(x)+\ddot{x}_{2 d}+d\left[\eta_{1}^{\prime} \arctan \left(K_{1} s_{1}\right)\right]\right\}$.

Substitute Eq. (31) into Eq. (34):

$$
\begin{aligned}
\dot{s}_{2}= & -[\hat{g}(u, x)-g(u, x)] u+\left[\widehat{f}_{2}(x)\right. \\
& \left.-f_{2}(x)\right]-\eta_{2}^{\prime} \arctan \left(K_{2} s_{2}\right)+d_{2}(t),
\end{aligned}
$$

Define $\tilde{\mathbf{W}}_{f_{2}}=\widehat{\mathbf{W}}_{f 2}-\mathbf{W}_{f 2}^{*}, \quad \tilde{\mathbf{W}}_{g}=\widehat{\mathbf{W}}_{g}-\mathbf{W}_{g}^{*}$.

Eq. (35) can be written as follows:

$$
\begin{aligned}
\dot{s}_{2} & =\tilde{\mathbf{W}}_{f_{2}} \mathbf{h}_{f_{2}}(x)-\varepsilon_{f_{2}}-\left[\tilde{\mathbf{W}}_{g} \mathbf{h}_{g}(x)-\varepsilon_{g}\right] u \\
& -\eta_{2}^{\prime} \arctan \left(K_{2} s_{2}\right)+d_{2}(t) .
\end{aligned}
$$

Take the Lyapunov function as:

$$
L_{2}=\frac{1}{2} s_{2}^{2}++\frac{1}{2 \gamma_{f_{2}}} \tilde{\mathbf{W}}_{f_{2}}^{T} \tilde{\mathbf{W}}_{f_{2}}+\frac{1}{2 \gamma_{g}} \tilde{\mathbf{W}}_{g}^{T} \tilde{\mathbf{W}}_{g} .
$$

The derivative of Eq. (37) is:

$$
\begin{aligned}
\dot{L}_{2} & =\tilde{\mathbf{W}}_{f_{2}}\left[s_{2} \mathbf{h}_{f_{2}}(x)-\frac{1}{\gamma_{f_{2}}} \dot{\widehat{\widehat{W}}}_{f_{2}}\right] \\
& -\tilde{\mathbf{W}}_{g}\left[s_{2} \mathbf{h}_{g}(x) u+\frac{1}{\gamma_{g}} \dot{\widehat{\mathbf{W}}}_{g}\right] \\
& +s_{2}\left[-\varepsilon_{f_{2}}+\varepsilon_{g} u-\eta_{2}^{\prime} \arctan \left(K_{2} s_{2}\right)+d_{2}(t)\right] .
\end{aligned}
$$

The adaptive law of $\tilde{\mathbf{W}}_{f_{2}}$ and $\mathbf{W}_{g}^{*}$ is:

$$
\left\{\begin{array}{l}
\dot{\widehat{\mathbf{W}}}_{f_{2}}=\gamma_{f_{2}} s_{2} \mathbf{h}_{f_{2}}(x) \\
\dot{\widehat{\mathbf{W}}}_{\phi_{2}}=-\gamma_{g} s_{2} \mathbf{h}_{g}(x) u
\end{array} .\right.
$$

Eq. (37) is written as follows:

$$
\begin{aligned}
\dot{L}_{2} & =s_{2}\left[-\varepsilon_{f_{2}}+\varepsilon_{g} u-\eta_{2}^{\prime} \arctan \left(K_{2} s_{2}\right)+d_{2}(t)\right] \\
& =s_{2}\left[-\varepsilon_{f_{2}}+\varepsilon_{g} u+d_{2}(t)\right]-\eta_{2}\left|s_{2}\right| .
\end{aligned}
$$

When the approximation error $\varepsilon_{f_{2}}$ and $\varepsilon_{g}$ is limited to a small enough range, if $\eta_{2}$ satisfies $\eta_{2} \geq \mid-\varepsilon_{f_{2}}+\varepsilon_{g} u+d_{2}(t)$, then $L_{2} \leq 0$. When $\dot{L}_{2} \equiv 0$, $s_{2} \equiv 0$. According to Lasalle's invariant set principle, the system is asymptotically convergent and globally stable. $t \rightarrow \infty, s_{2} \rightarrow 0$. Since $L_{2} \geq 0$ and $L_{2} \leq 0$, then when $t \rightarrow \infty, L_{2}$ is bounded.

\section{SIMULATION AND ANALYSIS}

A system simulation model is built in Simulink to verify the effectiveness of the proposed control 
strategy in this study [29]. The simulation parameters are listed in Table 1.

Table 1. System parameter

\begin{tabular}{cccc}
\hline No. & Symbol & Description & Values \\
\hline 1 & $A$ & Hydraulic area & $0.01 \mathrm{~m}^{2}$ \\
\hline 2 & $V_{0}$ & Initial volume of cavity & $0.00124 \mathrm{~m}^{3}$ \\
\hline 3 & $m$ & Total load & $2000 \mathrm{~kg}$ \\
\hline 4 & $B$ & Viscous damping coefficient & $95 \mathrm{~N} \cdot \mathrm{s} / \mathrm{m}$ \\
\hline 5 & $\rho$ & Oil density & $850 \mathrm{~kg} / \mathrm{m}^{3}$ \\
\hline
\end{tabular}

To prove the effectiveness of the new control strategy, a proportional-integral-derivative (PID) controller is compared [30].

1. The PID controller: $k_{p}=1500, k_{i}=10, k_{d}=0$;

2. RBF neural network sliding model control with extended state observer (RBFSMESO) (): $k_{1}=3$, $k_{2}=2, c_{e 1}=5, d_{e 1}=6, d_{e 2}=1, \gamma_{f_{2}}=12, \gamma_{g}=2.5$, $K_{1}=K_{2}=1000$.

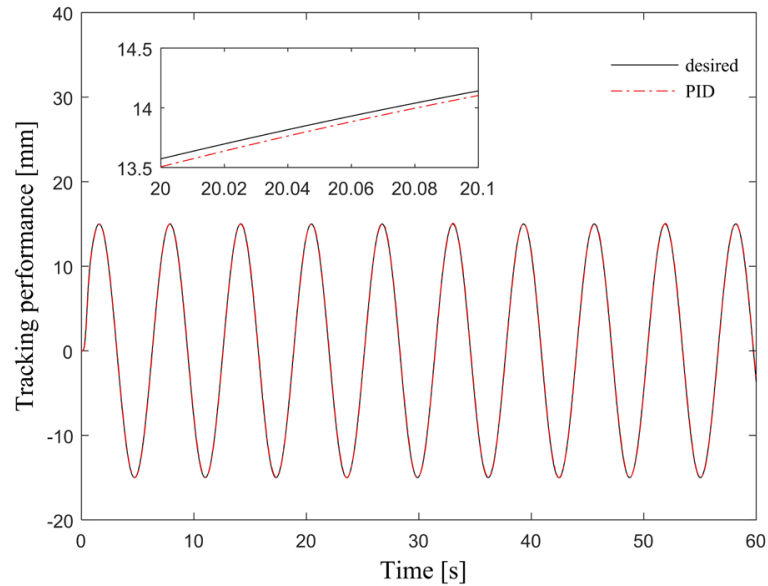

The simulation analysis of low-speed tracking and weak external interference, high-speed tracking and strong external interference is carried out below.

1. Low-speed tracking and weak external interference

Expected curve of trajectory tracking:

$$
x_{1 d}=0.015 \sin (0.159 \times 2 \pi t)\left[1-\exp \left(-10 t^{3}\right)\right] \text {, }
$$

and external load interference:

$$
\left.\begin{array}{c}
f_{d 1}=0 \quad t<5 \mathrm{~s} \\
f_{d 2}=20000\left\{1-\exp \left[-0.1 \times(t-5)^{3}\right]\right\} \quad t \geq 5 \mathrm{~s} \\
f_{d 3}=5000 \sin (2 \pi t) \quad t \geq 15 \mathrm{~s}
\end{array}\right\} .
$$

The track tracking performance and tracking error of PID and RBFSMESO controllers are shown in Figs. 5 and 6, respectively. It can be seen from the figures that the expected speed is slow (approximately $0.159 \mathrm{~Hz}$ ), and the external interference is small

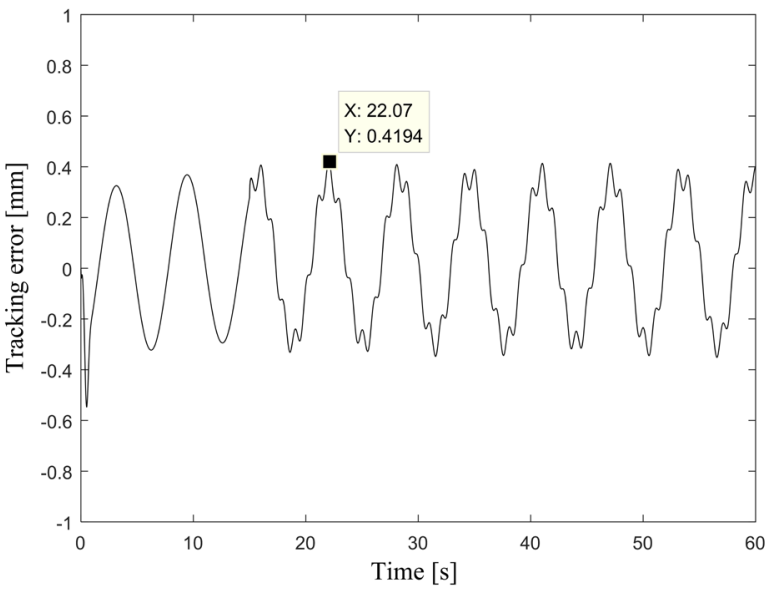

Fig. 5. PID tracking performance and tracking error
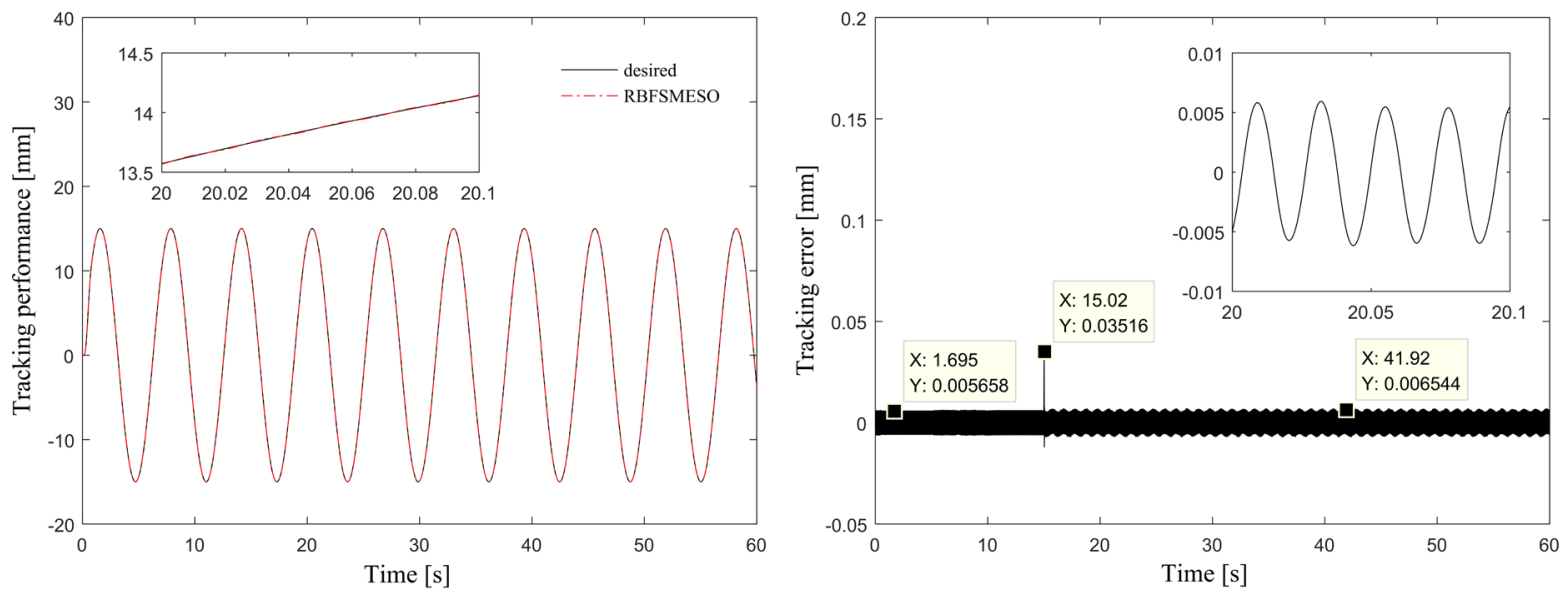

Fig. 6. Tracking curve of backstepping method 
$(20000 \mathrm{~N})$, so both of the two controllers have achieved good track tracking performance. However, RBFSMESO has better trajectory performance than the PID does due to the former's good model and external interference compensation capability. The maximum trajectory tracking error is no more than $0.006 \mathrm{~mm}$, while the maximum tracking error of the PID controller reaches $0.42 \mathrm{~mm}$.

At the same time, by comparing the tracking error curves of the two, it can be seen that the PID controller's tracking error significantly increases when constant external interference is added at $t \geq 5 \mathrm{~s}$, while the RBFSMESO's tracking error increases instantaneously, but with the accurate observation and compensation of external interference of the disturbance observer, its tracking error converges quickly. When $t \geq 15 \mathrm{~s}$, the PID controller has a large tracking error deformation after sinusoidal external interference is used, and external interference has no basically influence on the RBFSMESO controller, which further verifies the good anti-interference capability of the RBFSMESO controller.

Fig. 7 shows the observation performance and observation error of extended state observer (ESO) for external interference in RBFSMESO. When external disturbance of constant value is added at $t \geq 5 \mathrm{~s}$, the ESO observation error increases instantaneously. However, when external disturbance value tends to be stable, the observation error rapidly converges. The instantaneous maximum disturbance observation error is no more than $50 \mathrm{~N}$. When sinusoidal external interference is applied, the observation error increases due to the bandwidth limitation of the observer, but the maximum tracking error is still no more than $120 \mathrm{~N}$, which further verifies the good observation performance of the observer. Meanwhile, by comparing Figs. 6 and 7, it can be seen that the trajectory tracking error of RBFSMESO corresponds
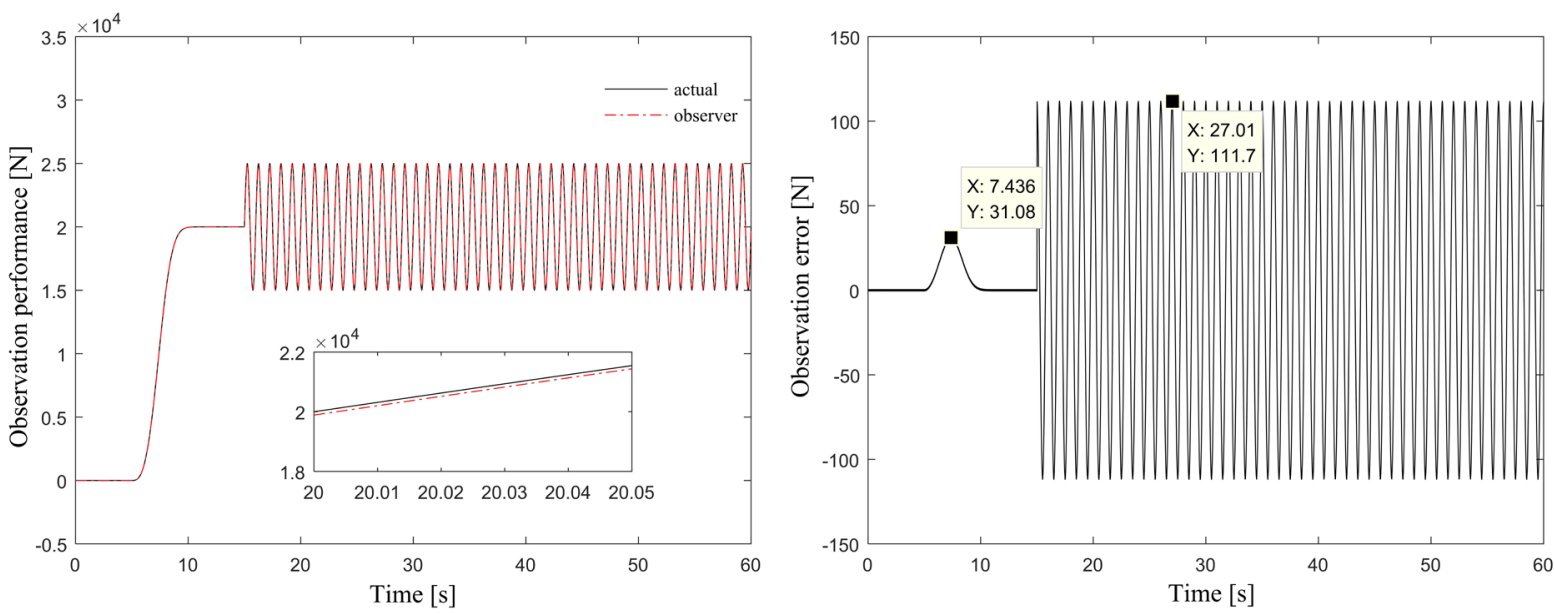

Fig. 7. The observation performance and observation error of ESO
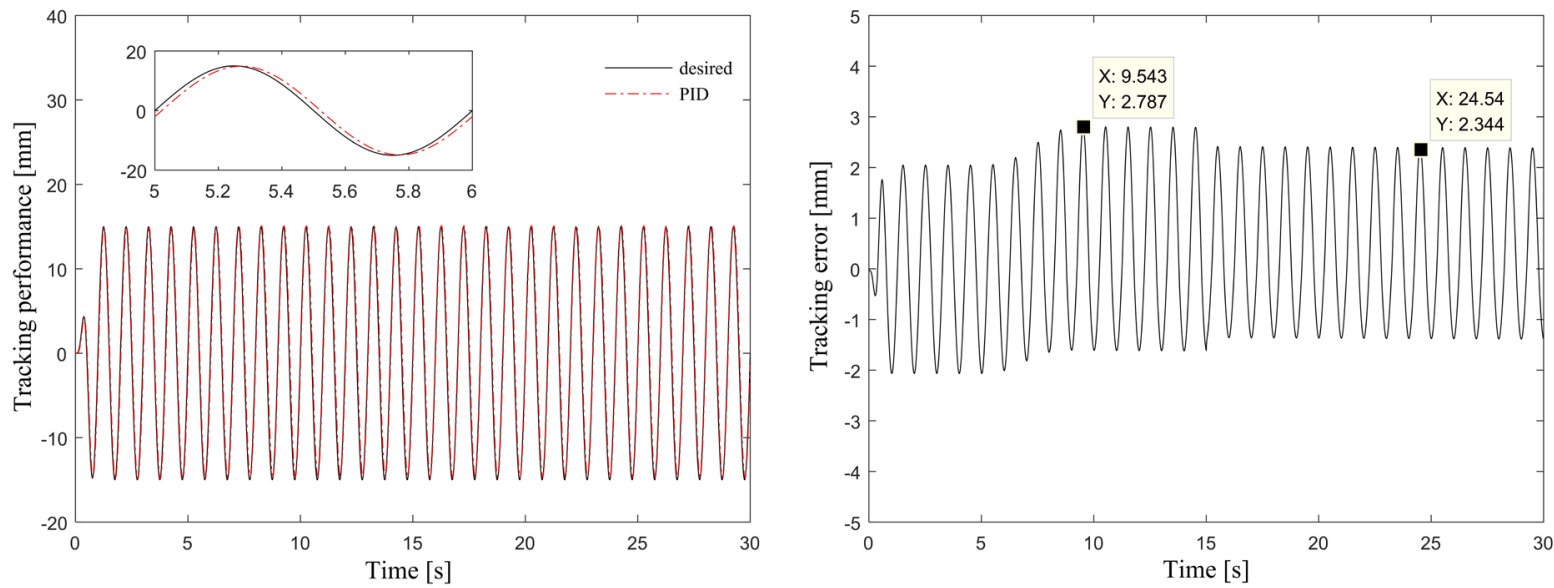

Fig. 8. PID trajectory-tracking performance and tracking error 
to the disturbance observation error of ESO, which verifies the importance of accurate disturbance observation and compensation to the controller performance.

2. High-speed tracking and strong external interference

Expected curve of trajectory tracking:

$$
x_{1 d}=0.015 \sin (2 \pi t)\left[1-\exp \left(-10 t^{3}\right)\right],
$$

and external load interference:

$$
\left.\begin{array}{c}
f_{d 1}=0 \quad t<5 \mathrm{~s} \\
f_{d 2}=120000\left\{1-\exp \left[-0.1 \times(t-5)^{3}\right]\right\} \quad t \geq 5 \mathrm{~s} \\
f_{d 3}=30000 \sin (2 \pi t) \quad t \geq 15 \mathrm{~s}
\end{array}\right\} .
$$

In the case of high-speed tracking and strong external interference, the trajectory-tracking performance and tracking error of the PID and
RBFSMESO controllers are shown in Figs. 8 and 9. By comparing with Figs. 5 and 6 , it can be seen that with the increase of interference, the trajectorytracking performance of the two controllers deteriorates to a certain extent, and the maximum tracking error reaches $2.8 \mathrm{~mm}$ and $0.0087 \mathrm{~mm}$ respectively. It can be found that RBFSMESO's trajectory tracking-performance is far better than PID due to its good anti-interference ability.

Fig. 10 shows the observation performance and observation error of ESO to external interference. The comparison with Fig. 7 shows that with the increase of external interference, the observation error also increases to a certain extent. However, compared with the maximum external interference of 150000 $\mathrm{N}$, the maximum observation error is $666 \mathrm{~N}$, which still shows the good observation performance of the observer.
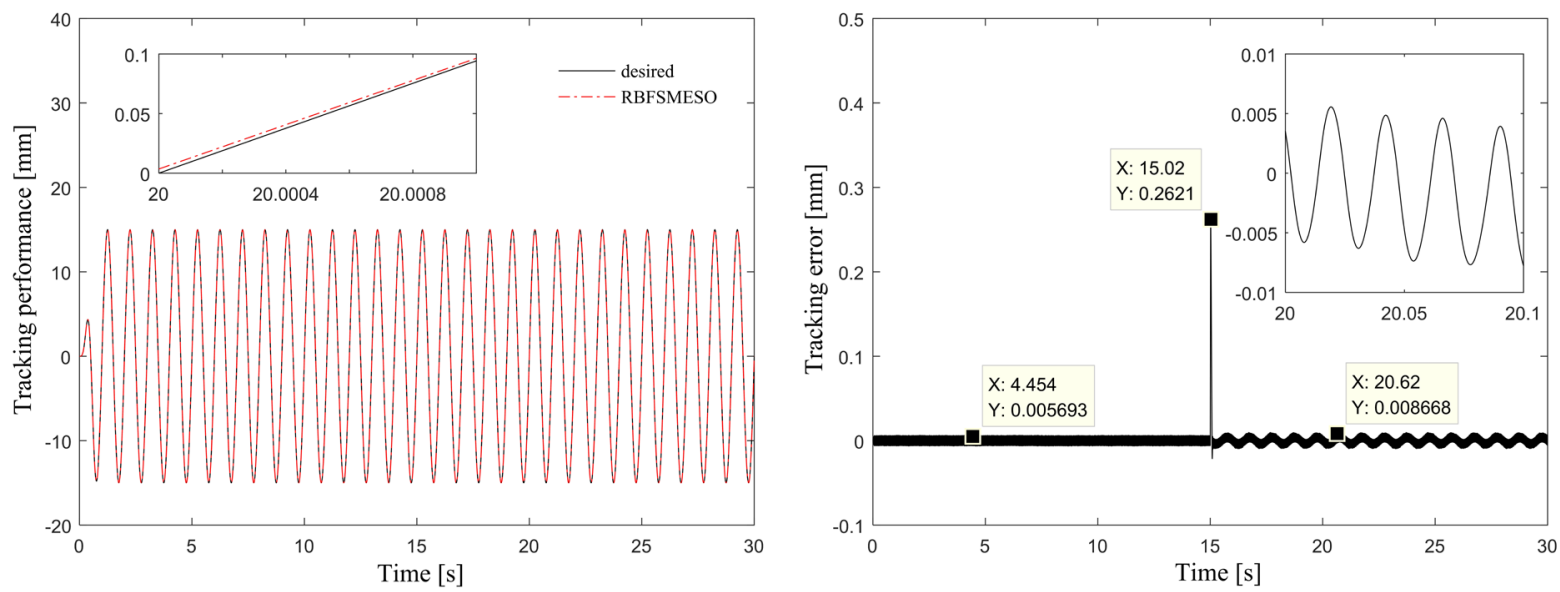

Fig. 9. Step response curve of backstepping method
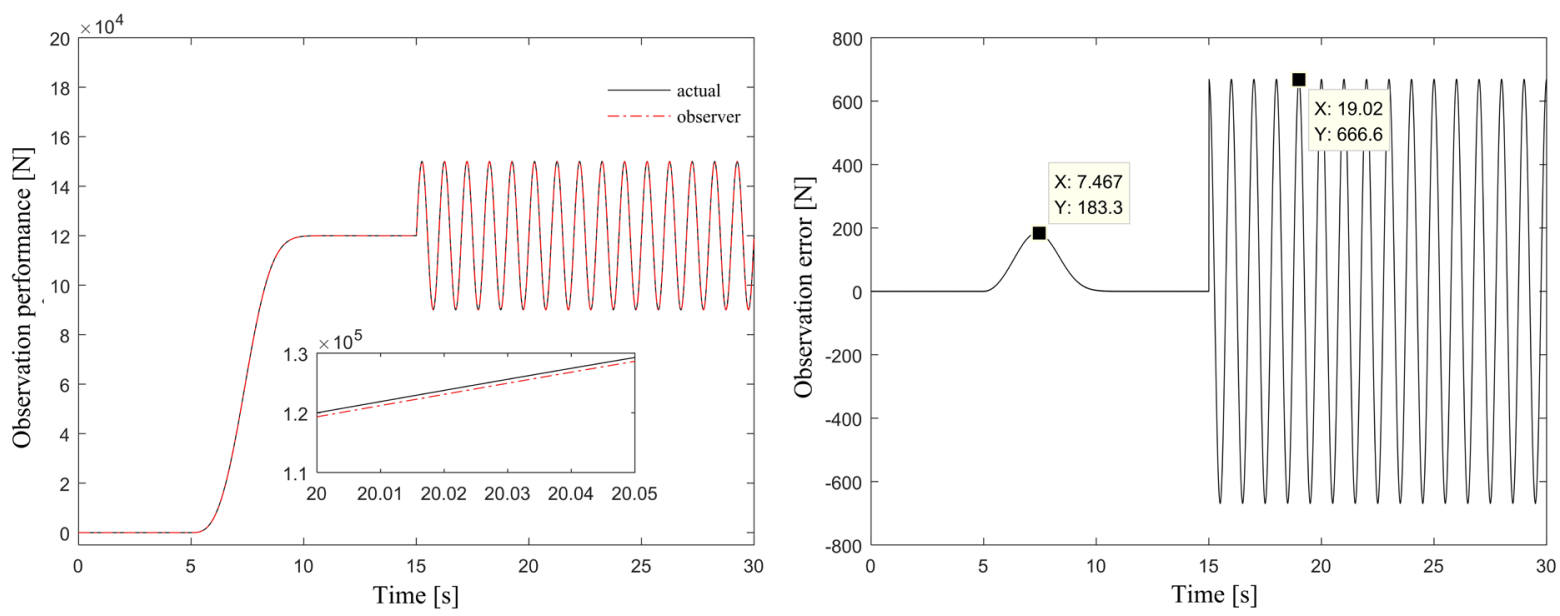

Fig. 10. The observation performance and observation error of ESO 


\section{EXPERIMENTAL VERIFICATION}

An experiment was carried out on the semi-physical experimental platform to verify further the feasibility of the strategy. The semi-physical experimental platform consists of an inertial load, a set of force loading equipment, a set of position measurement systems and a set of actuators testers. The inertial load is used to simulate the magnitude of the inertial force and the frictional damping. The force loading device can apply the predetermined force and can be used to simulate the disturbing force. The position measurement system can measure the position response of the load in real time. Because the connection is not completely stiff, the measured value will be different from the displacement output of the actuator itself in dynamic motion. The actuator tester is used for the instruction issuing of electro-hydraulic actuators and the detection and data analysis of each parameter. The displacement, velocity, and differential load pressure were collected via sensors [31].

1. Low-speed tracking condition

Given the following trajectory tracking expectation curve:

$$
x_{1 d}=0.015 \sin (0.159 \times 2 \pi t)\left[1-\exp \left(-10 t^{3}\right)\right] .
$$

The PID and RBFSMESO controllers are used to verify the trajectory tracking of the curve given above. The parameters of the controller are specified in Section 3.2. The trajectory-tracking performance and tracking error curves of the two controllers are shown in Figs. 11 and 12, respectively. The maximum trajectory tracking errors of the two controllers are $1.181 \mathrm{~mm}$ and $0.4567 \mathrm{~mm}$, respectively. Combined with the above simulation results, it can be seen that RBFSMESO further improves the trajectory tracking accuracy of the system through the function of RBF neural network and sliding mode control on
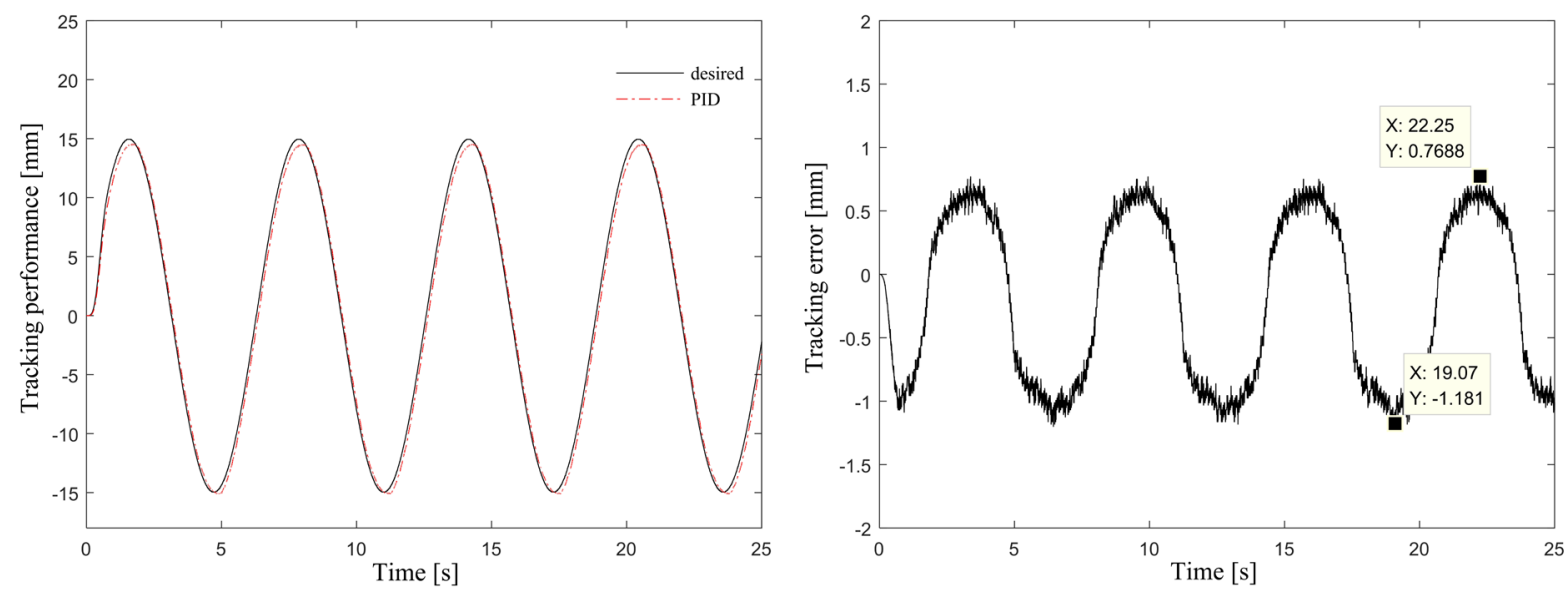

Fig. 11. PID trajectory-tracking performance and tracking error
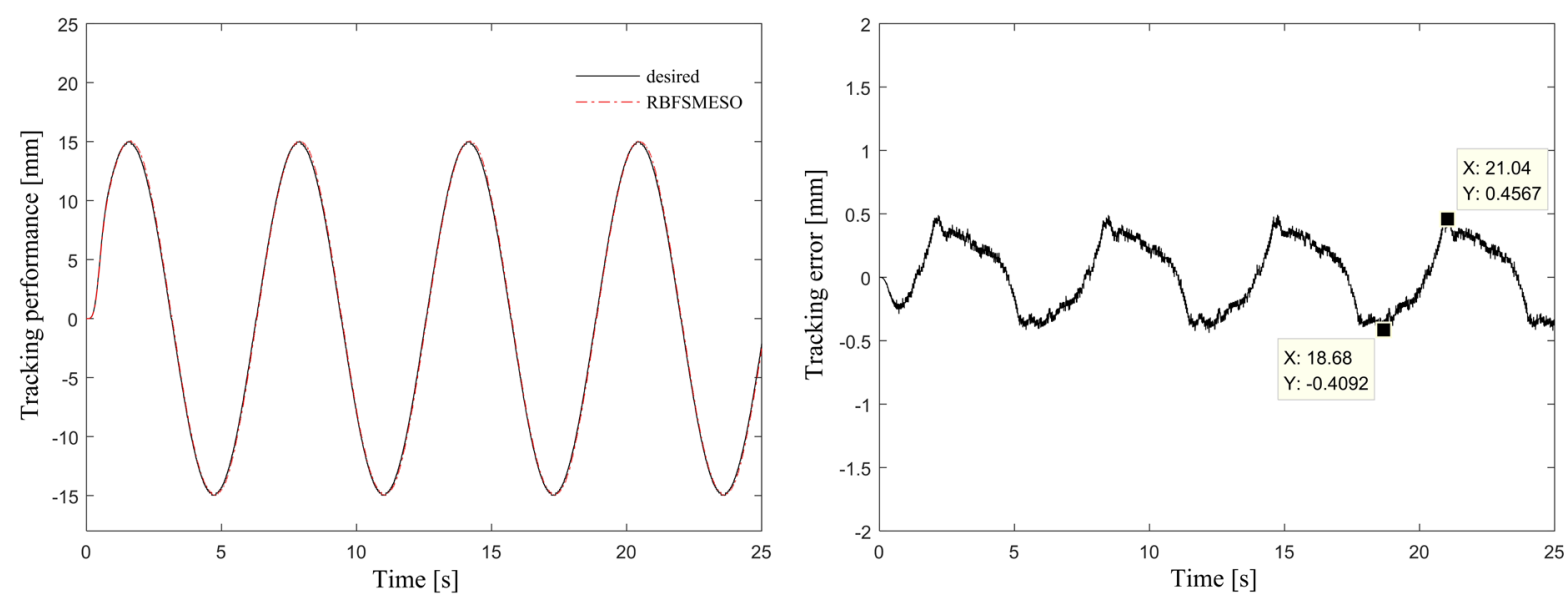

Fig. 12. RBFSMESO trajectory-tracking performance and tracking error 
the basis of introducing disturbance observation and compensation. However, the experimental error of the two controllers is greater than the simulation error, because the simulation model ignores some modelling error terms of the system, including the servo valve dynamics, the friction modelling error of the actuator and other unmodeled high-frequency dynamics, which leads to the increase of the trajectory tracking error of the controller.

2. High speed tracking condition

Expected curve of trajectory tracking:

$$
x_{1 d}=0.015 \sin (2 \pi t)\left[1-\exp \left(-10 t^{3}\right)\right] .
$$

PID and RBFSMESO controllers are still used to verify the trajectory tracking of the curve given above. The trajectory-tracking performance and tracking error of the PID and RBFSMESO controllers under high-speed tracking are shown in Figs. 13 and 14, respectively.
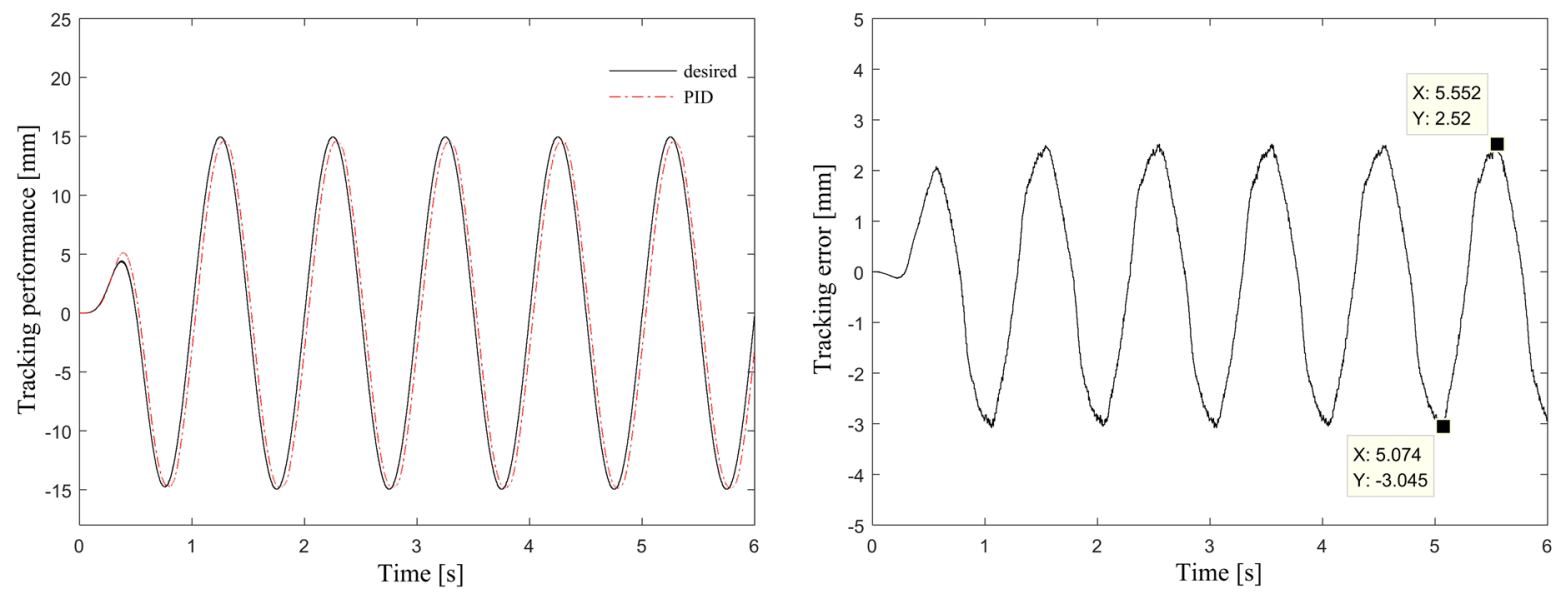

Fig. 13. PID trajectory-tracking performance and tracking error
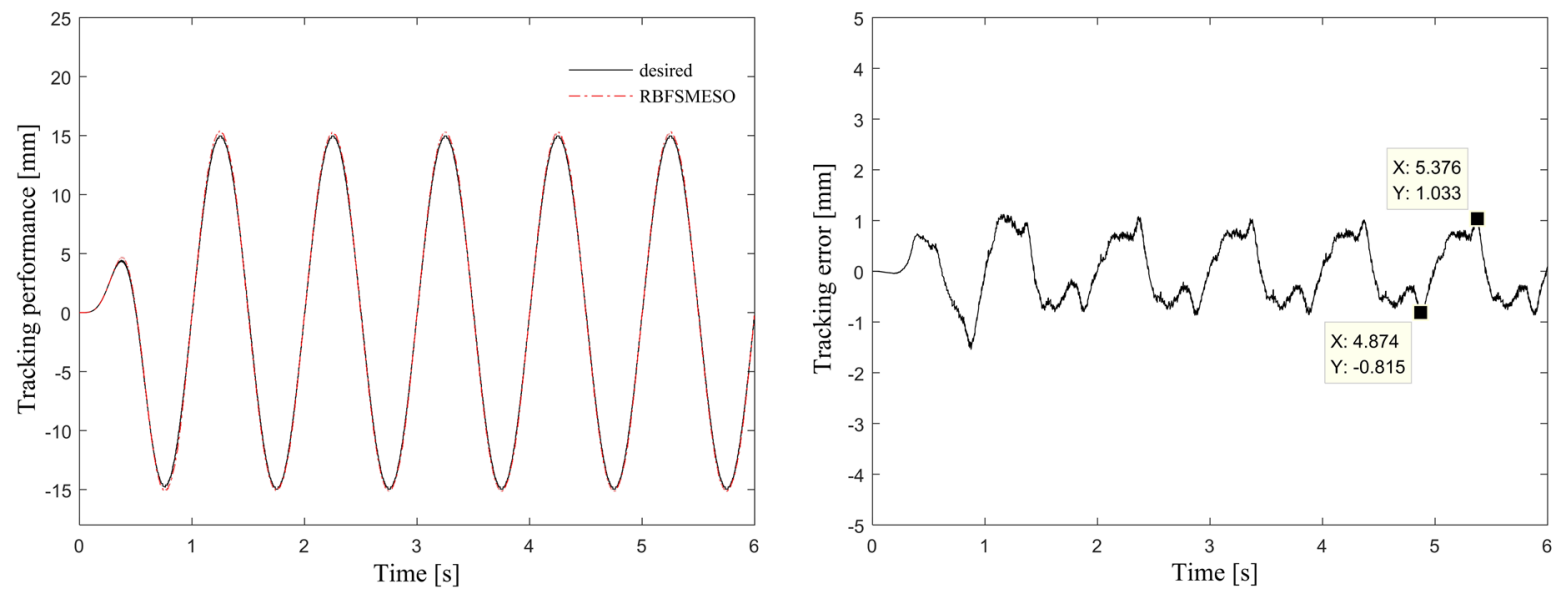

Fig. 14. RBFSMESO trajectory-tracking performance and tracking error 
on backstepping for an electro-hydraulic actuator is proposed. ESO is used to accurately estimate and compensate external disturbances, and the stability of the system is guaranteed by a robust control law. Simulation and experimental results show that ESO has good disturbance observation performance; the RBF sliding mode backstepping robust controller has good track tracking performance and has better tracking accuracy than traditional PID controller. The control strategy proposed in this paper improves the trajectory tracking accuracy and anti-jamming capability of the system, and shows strong robustness.

\section{REFERENCES}

[1] Ghazali, R., Paharudin, M., Sam, Y.M., Hussien, S.Y.S, Jali, M. (2017). Intelligent controller design for a non-linear quarter-car active suspension with electro-hydraulic actuator. Journal of Engineering Science and Technology, vol. 12, p. 39-51.

[2] Nguyen, M.T., Dang, T.D., Ahn, K.K. (2019). Application of electro-hydraulic actuator system to control continuously variable transmission in wind energy converter. Energies, vol. 12, no. 13, Dol:10.3390/en12132499.

[3] Li, W.D., Shi, G.L. (2019). Novel dual-redundancy electrohydrostatic actuator research and controller design. Proceedings of the Institution of Mechanical Engineers, Part C: Journal of Mechanical Engineering Science, vol. 233, no. 16, p. 5874-5886, DOI:10.1177/0954406219855093.

[4] Wang, W., Zhao, J., Ding, H. (2018). Output feedback non-linear energy-saving position control of electro-hydraulic asymmetric actuator. Proceedings of the Institution of Mechanical Engineers, Part l: Journal of Systems and Control Engineering, vol. 232, no. 3, p. 233-243, D0I:10.1177/0959651817746895.

[5] Baek, S.G., Ji, S., Koo, J.C. (2018). Experimental study of electro-hydraulic actuator with payload for precision motion control. Microsystem Technologies, vol. 24, p. 1347-1357, D0I:10.1007/s00542-016-3050-9.

[6] Li, D., Li, Y., Li, Y., Zhang, P., Dong, S., Yang, L. (2018). Study on PMSM Power Consumption of Dual-Variable ElectroHydraulic Actuator with Displacement-Pressure Regulation Pump. IEEE/ASME International Conference on Advanced Intelligent Mechatronics, p. 1172-1177, D0l:10.1109/ AIM.2018.8452426.

[7] Ali, S.A., Langlois, N. (2017). Sampled data's observer design with time varying correction gain for electro hydraulic actuator systems. American Control Conference, p. 3276-3281, DOI:10.23919/ACC.2017.7963452.

[8] Guo, K., Wei, J., Tian, Q. (2015). Non-linear adaptive position tracking of an electro-hydraulic actuator. Proceedings of the Institution of Mechanical Engineers, Part C: Journal of Mechanical Engineering Science, vol. 229, no. 17, p. 32523265, Dol:10.1177/0954406214568821.

[9] Alam, W., Mehmood, A., Ali, K., Javaid, U., Alharbi, S. Iqbal, J. (2018). Non-linear control of a flexible joint robotic manipulator with experimental validation. Strojniški vestnik Journal of Mechanical Engineering, vol. 64, no. 1, p. 47-55, DOI:10.5545/sv-jme.2017.4786.
[10] Wang, X.G., Li, L., Wei, X.L., Chen, G.Q., Liu, B.F. (2014). Electro-hydraulic servo actuator fuzzy self-tuning PID control research. Applied Mechanics and Materials, vol. 607, p. 795798, D0I:10.4028/www.scientific.net/amm.607.795.

[11] Wang, X.G., Li, L., Han, H.L., Wie, X.L., An, M.D., Liu, B.F. (2014). Electro-hydraulic servo actuator parameters self-tuning threedimensional fuzzy control research. Applied Mechanics and Materials, vol. 607, p. 811-814, D0l:10.4028/www.scientific. net/amm.607.811.

[12] Bellad, K., Hiremath, S.S., Singaperumal, M., Karunanidhi, S. (2014). Optimization of PID parameters in electro-hydraulic actuator system using genetic algorithm. Applied Mechanics and Materials, vol. 592-594, p. 2229-2233, D0l:10.4028/ www.scientific.net/amm.592-594.2229.

[13] Németh, B., Varga, B., P. Gáspÿr, P. (2014). Robust control design of an electro-hydraulic actuator. IEEE/ ASME International Conference on Advanced Intelligent Mechatronics, p. 245-250, D0I:10.1109/AIM.2014.6878086.

[14] Ling, T.G., Rahmat, M.F., Husain, A.R. (2014). ANFIS modeling of electro-hydraulic actuator system through physical modeling and FCM gap statistic in initial FIS determination. Journal of Intelligent \& Fuzzy Systems, vol. 27, no. 4, p.1743-1755, DOI:10.3233/IFS-141140.

[15] Javaid, U., Mehmood, A., Arshad, A., Imtiaz, F., lqbal, J. (2020). Operational efficiency improvement of PEM fuel cell-A sliding mode based modern control approach. IEEE Access, vol. 8, p. 95823-95831, DOI:10.1109/ACCESS.2020.2995895.

[16] Wrat, G., Ranjan, P., Bhola, M., Mishra, S.K., Das, J. (2019). Position control and performance analysis of hydraulic system using two pump-controlling strategies. Proceedings of the Institution of Mechanical Engineers, Part I: Journal Systems and Control Engineering, vol. 233, no. 9, p. 1093-1105, DOI:10.1177/0959651818813233.

[17] Yang, G.C., Yao, J.Y. (2019). Output feedback control of electro-hydraulic servo actuators with matched and mismatched disturbances rejection. Journal of the Franklin Institute, vol. 356, no. 16, p. 9152-9179, D0l:10.1016/j. jfranklin.2019.07.032.

[18] Németh, B., Fényes, D., Gáspár, P., Bokor, J. (2017). Control design of an electro-hydraulic actuator for variable-geometry suspension systems. 25th Mediterranean Conference on Control and Automation, p. 180-185, D0l:10.1109/ MED.2017.7984115.

[19] Guo, Q., Yu, T., Jiang, Dan, J. (2015). Adaptive backstepping design of electro-hydraulic actuator based on state feedback control. International Conference on Fluid Power and Mechatronics, p. 888-891, D0I:10.1109/FPM.2015.7337239.

[20] Liu, Y., Zhao, Z. Guo, F. (2016). Adaptive Lyapunov-based backstepping control for an axially moving system with input saturation. IET Control Theory and Applications, vol. 10, no. 16, p. 2083-2092, D0l:10.1049/iet-cta.2016.0417.

[21] Echeikh, H., Trabelsi, R., Iqbal, A., Bianchi, N., Mimouni, M.F. (2016). Non-linear backstepping control of five-phase IM drive at low speed conditions-experimental. ISA Transactions, vol. 65, p. 244-253, D0I:10.1016/j.isatra.2016.06.013.

[22] Liem, D.T., Truong, D.Q., Park, H.G., Ahn, K.K. (2016). A Feedforward neural network fuzzy grey predictor-based controller for force control of an electro-hydraulic actuator. 
International Journal of Precision Engineering and Manufacturing, vol. 17, p. 309-321, D0I:10.1007/s12541016-0039-3.

[23] Zhou, H., Lao, L., Chen, Y., Yang, H. (2017). Discrete-time sliding mode control with an input filter for an electro-hydraulic actuator. IET Control Theory and Applications, vol. 11, no. 9, p. 1333-1340, D0I:10.1049/iet-cta.2016.0951.

[24] Loukianov, A.G., Sanchez, E., Lizalde, C. (2008). Force tracking neural block control for an electro-hydraulic actuator via second-order sliding mode. International Journal of Robust And Nonlinear Control, vol. 18, no. 3, p. 319-332, DOl:10.1002/rnc.1222.

[25] Gao, Z.Q. (2003). Scaling and bandwidth-parameterization based controller tuning. Proceedings of the American Control Conference, p. 4989-4996, D0I:10.1109/ACC.2003.1242516.
[26] Yang, Y., Zhao, D., Ma, L., Zhu, Q., Huang, D. (2016). Backstepping Trajectory Tracking Control of Electro-Hydraulic Actuators of Lower Limb Load Exoskeleton. 42nd Annual Conference of the IEEE-Industrial-Electronics-Society. p. 60736078, DOI:10.1109/IECON.2016.7793005.

[27] Nahian, S.A., Truong, D.Q., Chowdhury, P., Das, D., Ahn, K.K. (2016). Modeling and fault tolerant control of an electrohydraulic actuator. International Journal of Precision Engineering and Manufacturing, vol. 17, p.1285-1297, DOI:10.1007/s12541-016-0153-2.

[28] Ioannou, P.A. Sun, J. (1996). Robust Adaptive Control. Prentice-Hall, Upper Saddle River, p. 75-76.

[29] Liang, L.X., Faudzi, A.A.M., Ismail, Z.H (2019). System identification and model predictive control using CVXGEN for electro-hydraulic actuator. International Journal of Integrated Engineering, vol. 11, no. 4, p. 166-174. 